\title{
A DFT Investigation of the Molecular Structure and UV Absorption Spectrum of 2-Ethylhexyl 2-Hydroxybenzoate (Octisalate) and Meta-Substituted 2-Ethylhexyl 2-Hydroxybenzoate: Sunscreen Applications
}

\author{
Wikorn Punyain* \\ Department of Chemistry and NU-Research Center for Petroleum, Petrochemicals and Advanced \\ Materials, Faculty of Science, Naresuan University, Phitsanulok, 65000 Thailand \\ e-mail: kraiwanp@nu.ac.th
}

\begin{abstract}
Keywords: Sunscreen, Density Functional Theory (DFT), 2-ethylhexyl 2-hydroxybenzoate, UV spectrum
\end{abstract}

\begin{abstract}
. 2-ethylhexyl 2-hydroxybenzoate (octisalate) is one of organic compounds containing in sunscreen products to absorb ultraviolet radiation. Density Functional Theory (DFT) was used to investigate the molecular structure and the ultraviolet (UV) absorption spectrum of 2-ethylhexyl 2-hydroxybenzoate and meta-substituted 2-ethylhexyl 2-hydroxybenzoate to model the novel sunscreen compounds. The geometry optimizations and frequency calculations were done at B3LYP/6-311++G(d,p) level of theory. The 10 vertical excitation calculations were performed by Time-Dependent Density Functional Theory (TD-DFT) at B3LYP/6-311++G(d,p) level. The solvent effects were taken into account by using the Polarizable Continuum Model (CPCM). It was found that the intramolecular hydrogen bond occurred in the 2-ethylhexyl 2-hydroxybenzoate structure. The UV absorption spectrum in the UVA and UVB regions are $\pi \rightarrow \pi^{*}$ transitions (HOMO $\rightarrow$ LUMO transitions). The calculated UV absorption spectrum of the 2-ethylhexyl 2-hydroxybenzoate and its derivatives are significantly affected by the substitution groups.
\end{abstract}

\section{Introduction}

Although ozone in the atmosphere absorbs UV radiation from the sun, but in fact it can absorb only UVC region (200-280 nm). From the scientific reports, UVA (320-400 nm) and UVB (280$320 \mathrm{~nm}$ ) radiation cause sunburn, skin damaging and skin cancer [1-2]. Several organic UV absorbing compounds were synthesized and developed continuously from the past decade until nowadays for using in sunscreen products and UV filter materials. There are several sunscreen products brought to the market around the world from the past decade until now for protecting body from skin damage [3-6]. Octisalate (2-ethylhexyl 2-hydroxybenzoate) is one of the organic UVB absorbing compounds which widely used in the composition of sunscreen and cosmetic products [7-8].

Nowadays several research groups investigate various organic UV absorbing compounds for the sunscreen applications by theoretical studies and experiments [9]. T.Kartrick and coworkers performed experimental and theoretical studies of FT-IR and FT-Raman spectra of 5-nitrosalisylic acid which has three different substitution groups (hydroxyl group $(-\mathrm{OH})$, carboxyl group $(-\mathrm{COOH})$, and nitro group $\left(-\mathrm{NO}_{2}\right)$ ). It was found that the vibrations of carboxylic and hydroxyl groups in 5-nitrosalisylic acid are involved in the interaction of intra and intermolecular hydrogen bonds [10]. Bianca A.M. Correa and coworkers investigated and modeled the UV absorption spectra of benzophenone derivatives by Density Functional Theory (DFT) and Time-Dependent Density Functional Theory (TD-DFT). It was found that the main electronic transition of benzophenone derivatives are in the UVA and UVB range which result from the $\pi \rightarrow \pi^{*}$ transitions (HOMO $\rightarrow$ LUMO). They are also mention that benzophenone derivatives with protic substitution groups at para position showed the absorption in UVB range while at ortho position presented the UVA absorption region [11]. 
This present work, we focuses on the theoretical photo-physics investigations (molecular structure and UV absorption spectrum) of 2-ethylhexyl 2-hydroxybenzoate and its derivatives to see the effect on UVA and UVB absorption spectrum from substitution groups (electron withdrawing groups and electron donor groups) at meta position. Another one aspect is the influence of the strength of the electron donating groups to the UVA and UVB absorption range. The results from this work can be apply for the synthesize novel organic UV absorbing compounds to use in the composition of sunscreen products and UV filter materials which can absorb all UVA and UVB regions with high intensity.

\section{Method of calculations}

The starting geometries of two possible conformers of 2-ethylhexyl 2-hydroxybenzoate were constructed as shown in Fig. 1.

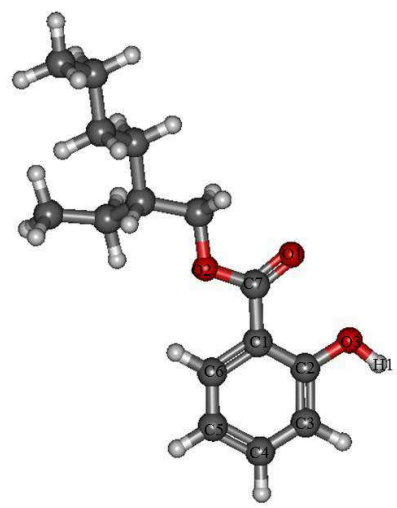

C1

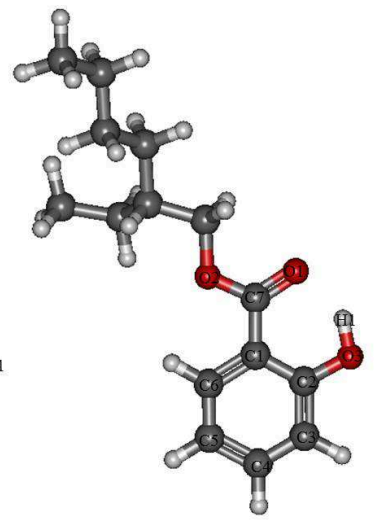

$\mathrm{C} 2$

Fig. 1. The optimized molecular structure of both two possible conformers of 2-ethylhexyl 2-hydroxybenzoate.

Density Functional Theory (DFT) was used to investigate in these calculations. The starting molecular geometries of 2-ethylhexyl 2-hydroxybenzoate were performed geometry optimizations in gas phase at B3LYP/6-311++G(d,p) level of theory. The frequency calculations were performed to the optimized geometries to verify that the optimized geometries are located on the local minima with no imaginary frequencies. Boltzmann statistics calculations were performed to all two possible conformers by using the following equation to find the most stable conformer and percent abundant.

$$
\frac{N_{i}}{N}=\frac{e^{\frac{-\epsilon_{i}}{k T}}}{\sum_{i} e^{\frac{-\epsilon_{i}}{k T}}}
$$

The 10 vertical excitation calculations were performed on both two gas phase optimized geometries with Time-Dependent Density Functional Theory (TD-DFT) at B3LYP/6-311++G(d,p) level of theory.

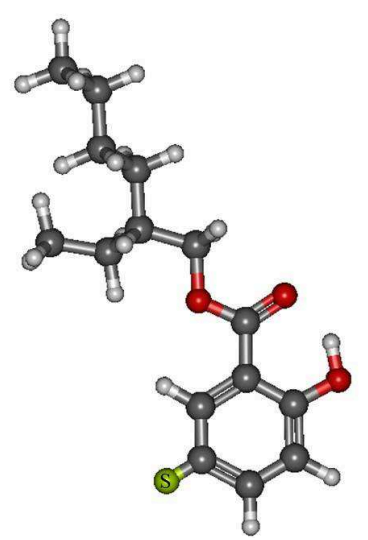

Fig. 2. The molecular geometries of substituted 2-ethylhexyl 2-hydroxybenzoate. 
The substitution groups, electron withdrawing groups $\left(\mathrm{CN}, \mathrm{NO}_{2}, \mathrm{~F}, \mathrm{Cl}, \mathrm{Br}\right)$ and electron donor groups $\left(\mathrm{NH}_{2}, \mathrm{NHCH}_{3}, \mathrm{~N}\left(\mathrm{CH}_{3}\right)_{2}, \mathrm{OH}, \mathrm{OCH}_{3}\right)$, were added to the most stable conformer of 2-ethylhexyl 2-hydroxybenzoate at meta position then performed geometry optimizations and frequency calculations follow by 10 vertical excitation at TD-DFT B3LYP/6-311++G(d,p) level to see the effect of the substitution groups for the UV absorption spectrum in the UVA and UVB regions. The solvent effects were taken into account by using the Polarizable Continuum Model (PCM) in methanol and water solvents. All of the calculations were done by using Gaussian09 program package [12] and Gabedit program package [13] for the molecular visualization.

\section{Results and Discussions}

The optimized molecular geometries at B3LYP/6-311++G(d,p) level of both two conformers of 2-ethylhexyl 2-hydroxybenzoate are located on the local minimum with no imaginary frequency which shown in figure 1 and the structural parameters were shown in Table 1.

Table 1. The structural parameters of the optimized structure 2-ethylhexyl 2-hydroxybenzoate in gas phase.

\begin{tabular}{lllllllll}
\hline Octisalate & \multicolumn{7}{c}{ Bond lengths/ $\AA$} \\
\cline { 2 - 9 } & $\mathrm{C} 1-\mathrm{C} 2$ & $\mathrm{C} 2-\mathrm{C} 3$ & $\mathrm{C} 3-\mathrm{C} 4$ & $\mathrm{C} 4-\mathrm{C} 5$ & $\mathrm{C} 5-\mathrm{C} 6$ & $\mathrm{C} 6-\mathrm{C} 1$ & $\mathrm{C} 1-\mathrm{C} 7$ & $\mathrm{C} 2-\mathrm{O} 3$ \\
\hline Conformer1 & 1.41 & 1.40 & 1.39 & 1.40 & 1.39 & 1.41 & 1.49 & 1.39 \\
Conformer2 & 1.42 & 1.41 & 1.39 & 1.41 & 1.39 & 1.41 & 1.47 & 1.35 \\
\hline \multicolumn{7}{c}{ Table 1. Continued. } \\
\hline Octisalate & \multicolumn{7}{c}{ Bond angles/ ${ }^{\circ}$} \\
\cline { 2 - 8 } & $\mathrm{C} 1-\mathrm{C} 7-\mathrm{O} 1$ & $\mathrm{C} 1-\mathrm{C} 7-\mathrm{O} 2$ & $\mathrm{C} 2-\mathrm{O} 3-\mathrm{H}$ & H-O3-C2-C1 \\
\hline Conformer1 & 126 & 112 & 109 & 180 & \\
Conformer2 & 124 & 114 & 107 & 0 & Dihedral angles/ \\
\hline
\end{tabular}

It was found that the carbon-carbon bond lengths in the benzene ring of both two conformers are around 1.4 $\AA$. The intramolecular hydrogen bond is occurred in the conformer 2 as show in Fig. 3 with the interatomic distance between $\mathrm{H}(1)-\mathrm{O}(1) 1.73 \AA$. The intramolecular hydrogen bond results in the energy of conformer 2 lower than conformer 1 as $49.45 \mathrm{~kJ} / \mathrm{mol}$. The Boltzmann distribution reveals that conformer 2 is the molecular structure of 2-ethylhexyl 2-hydroxybenzoate with $100 \%$ abundant as shown in Table 2.

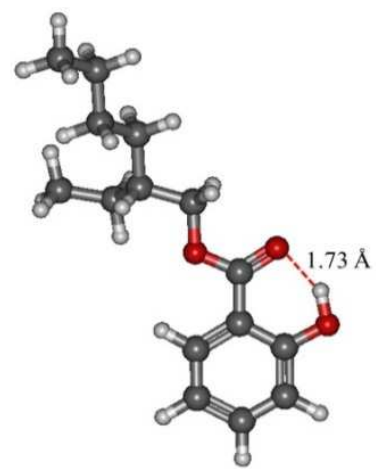

Fig. 3. The optimized structure of 2-ethylhexyl 2-hydroxybenzoate conformer 2.

Table 2. The relative energy and percent Boltzmann distribution of 2-ethylhexyl 2-hydroxybenzoate both two conformer in gas phase.

\begin{tabular}{lcc}
\hline Compounds & Relative Energy $/(\mathrm{kJ} / \mathrm{mol})$ & \% Boltzmann distribution \\
\hline Conformer1 & 49.45 & 0 \\
Conformer2 & 0 & 100 \\
\hline
\end{tabular}

TD-DFT calculations at B3LYP/6-311++G(d,p) level show the calculated UV absorption spectrum of 2-ethylhexyl 2-hydroxybenzoate both two conformers in gas phase (Fig. 4). It was found that both two conformers show three absorption peaks in the UV region $(200-400 \mathrm{~nm})$. The first absorption peak located at $207.79 \mathrm{~nm}$ for conformer1 and $209.58 \mathrm{~nm}$ for conformer2 which is 
charge transfer transitions (HOMO-3 $\rightarrow$ LUMO and HOMO $\rightarrow$ LUMO +1 for conformer1 and conformer2, respectively). The second peak at $230.46 \mathrm{~nm}$ (HOMO-2 $\rightarrow$ LUMO) for conformer1 and $237.14 \mathrm{~nm}$ (HOMO-1 $\rightarrow$ LUMO) for conformer2 are $\pi \rightarrow \pi^{*}$ transitions. The third peak at $276.83 \mathrm{~nm}$ and $296.64 \mathrm{~nm}$ for conformer1 and conformer2 are $\pi \rightarrow \pi^{*}$ transitions (HOMO $\rightarrow$ LUMO), see Table 3. The calculated absorption spectra of conformer2 are bathochromic shift (red shift) from conformerl for those three absorption peaks. When compared the calculated UV absorption spectrum of both two conformers to the experimental data $(307 \mathrm{~nm})$ [7], it was found that the UV absorption spectrum of conformer 2 corresponding well to the experiment.

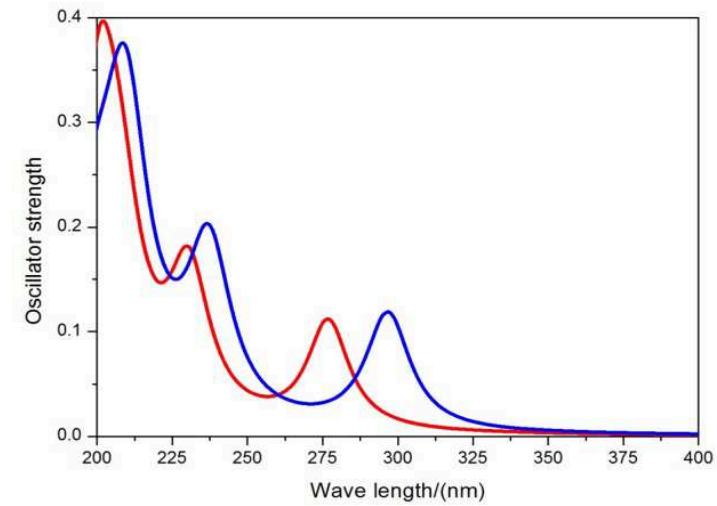

Fig. 4. The UV absorption spectrum of 2-ethylhexyl 2-hydroxybenzoate both conformer1 (red) and conformer2 (blue) in gas phase.

Table 3. The UV absorption peak and the electronic transitions of 2-ethylhexyl 2-hydroxybenzoate in gas phase.

\begin{tabular}{|c|c|c|c|}
\hline \multirow[t]{2}{*}{ Octisalate } & \multicolumn{2}{|c|}{ Absorption peak/(nm) } & Electronic transitions \\
\hline & Calc. & Exp. & \\
\hline \multirow[t]{6}{*}{ Conformer1 } & 207.79 & \multirow{6}{*}{307 [7] } & \\
\hline & & & HOMO-3 $\rightarrow$ LUMO \\
\hline & 230.46 & & \\
\hline & & & HOMO-2 $\rightarrow$ LUMO \\
\hline & 276.83 & & \\
\hline & & & HOMO $\rightarrow$ LUMO \\
\hline \multirow[t]{5}{*}{ Conformer2 } & 209.58 & \multirow[b]{5}{*}{307 [7] } & \\
\hline & & & $\mathrm{HOMO} \rightarrow \mathrm{LUMO}+1$ \\
\hline & 237.14 & & \\
\hline & & & HOMO-1 $\rightarrow$ LUMO \\
\hline & 296.64 & & HOMO $\rightarrow$ LUMO \\
\hline
\end{tabular}


The effects of substitution groups at meta position, electron withdrawing groups $\left(\mathrm{CN}, \mathrm{NO}_{2}, \mathrm{~F}\right.$, $\mathrm{Cl}, \mathrm{Br})$ and electron donor groups $\left(\mathrm{NH}_{2}, \mathrm{NHCH}_{3}, \mathrm{~N}\left(\mathrm{CH}_{3}\right)_{2}, \mathrm{OH}, \mathrm{OCH}_{3}\right)$, and effect of solvents (methanol, acetone, water) were taken into account at B3LYP/6-311++G(d,p) level. The calculated absorption spectrum from TD-DFT calculations in the UVA and UVB regions (third peak) of all compounds result from the $\pi \rightarrow \pi^{*}$ transitions (HOMO $\rightarrow$ LUMO), presented in Fig. 3 and Table 4. The absorption spectrum of 2-ethylhexyl 2-hydroxybenzoate substituted by the electron withdrawing groups are bathochromic shift from 2-ethylhexyl 2-hydroxybenzoate spectrum and located in the UVB region $(280-320 \mathrm{~nm})\left(\mathrm{CN}<\mathrm{NO}_{2}<\mathrm{F}<\mathrm{Cl}<\mathrm{Br}\right)$ while electron donor groups result in the absorption spectrum located in the UVA region $(320-400 \mathrm{~nm})$ $\left(\mathrm{OH}<\mathrm{OCH}_{3}<\mathrm{NH}_{2}<\mathrm{NHCH}_{3}<\mathrm{N}\left(\mathrm{CH}_{3}\right)_{2}\right)$ and the absorption intensity of 2-ethylhexyl 2hydroxybenzoate substituted by $\mathrm{NO}_{2}$ is approximately twice times when compared to other compounds (see Fig. 5). It is imply that the bathochromic shift in UVA and UVB regions for metasubstituted-2-ethylhexyl 2-hydroxybenzoate depends on the strength of electron donating groups. Moreover the UV absorption spectrum of the 2-ethylhexyl 2-hydroxybenzoate and its derivatives are not affected by the selected solvents (methanol and water) as shown in Table 4.

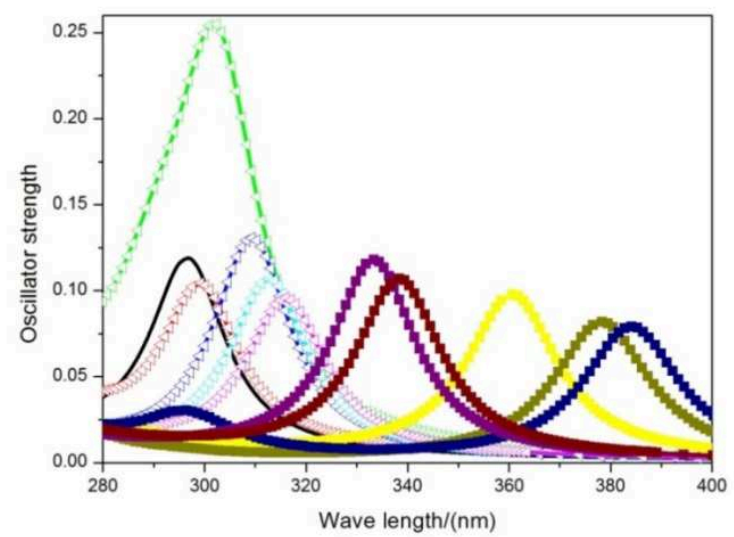

Fig. 5. The UV absorption spectrum in gas phase of 2-ethylhexyl 2-hydroxybenzoate (line) and its derivatives with substitution groups (electron donor groups (square) and electron withdrawing groups (triangle)). Electron withdrawing groups $\mathrm{CN}$ (red), $\mathrm{NO}_{2}$ (green), $\mathrm{F}$ (blue), $\mathrm{Cl}(\mathrm{cyan}$ ), $\mathrm{Br}$ (magenta) and electron donor groups $\mathrm{NH}_{2}$ (yellow), $\mathrm{NHCH}_{3}$ (dark yellow), $\mathrm{N}\left(\mathrm{CH}_{3}\right)_{2}$ (navy), $\mathrm{OH}$ (purple), $\mathrm{OCH}_{3}$ (wine).

Table 4. The absorption peak in the UVA and UVB regions of 2-ethylhexyl 2-hydroxybenzoate and its derivatives in gas phase, methanol and water.

\begin{tabular}{|c|c|c|c|}
\hline \multirow[t]{2}{*}{ Compounds } & \multicolumn{3}{|c|}{ Absorption spectrum/(nm) } \\
\hline & Gas phase & Methanol & Water \\
\hline 2-ethylhexyl 2-hydroxybenzoate & 296.64 & 296.42 & 296.37 \\
\hline$m$-CN-2-ethylhexyl 2-hydroxybenzoate & 299.21 & 294.22 & 294.09 \\
\hline$m$-NO ${ }_{2}$-2-ethylhexyl 2-hydroxybenzoate & 332.07 & 330.80 & 330.78 \\
\hline$m$-F-2-ethylhexyl 2-hydroxybenzoate & 309.34 & 307.73 & 307.65 \\
\hline$m$-Cl-2-ethylhexyl 2-hydroxybenzoate & 312.74 & 310.35 & 310.26 \\
\hline$m$-Br-2-ethylhexyl 2-hydroxybenzoate & 316.05 & 313.31 & 313.22 \\
\hline$m$-NH 2 -2-ethylhexyl 2-hydroxybenzoate & 360.72 & 366.96 & 367.09 \\
\hline$m$-NHCH 3 -2-ethylhexyl 2-hydroxybenzoate & 378.33 & 388.92 & 389.17 \\
\hline$m$-N $\left(\mathrm{CH}_{3}\right)_{2}$-2-ethylhexyl 2-hydroxybenzoate & 384.20 & 400.57 & 400.98 \\
\hline$m$-OH-2-ethylhexyl 2-hydroxybenzoate & 333.40 & 334.36 & 334.35 \\
\hline$m$-OCH 3 -2-ethylhexyl 2-hydroxybenzoate & 338.37 & 340.61 & 340.63 \\
\hline
\end{tabular}




\section{Conclusions}

In this work, we report two conformers of 2-ethylhexyl 2-hydroxybenzoate in gas phase. The calculational results show that conformer2 is the most stable conformer results from the intramolecular hydrogen bond. Density Functional Theory (DFT) and Time-Dependent Density Functional Theory (DFT) at B3LYP/6-311++G(d,p) level of theory are appropriate methods for the geometry optimizations and vertical excitation calculations yielding the calculational results corresponding well with the experimental results. The 2-ethylhexyl 2-hydroxybenzoate shows three peaks in UV absorption spectrum, two peaks located in UVC region and one peak located in UVB region. The absorption peak that located in UVB region result from the $\pi \rightarrow \pi^{*}$ transitions (HOMO $\rightarrow$ LUMO). The meta-substituted of 2-ethylhexyl 2-hydroxybenzoate presented the bathochromic shift of absorption spectrum from 2-ethylhexyl 2-hydroxybenzoate in the UVA and UVB regions. The bathochromic shift in the meta-sustituted 2-ethylhexyl benzoate is depends on the strength of electron donating groups. The calculated UV absorption spectrum of the 2ethylhexyl 2-hydroxybenzoate and its derivatives are not significantly affected by the solvents.

\section{Acknowledgements}

The authors acknowledge the research grant from Naresuan University and National e-Science Infrastructure Consortium for providing computing resources that have contributed to the research results reported within this paper.

\section{References}

[1] F.P. Gasparro, M. Mitchnick, J.F. Nash, A review of sunscreen safety and efficacy, Photochem. Photobiol. 68 (1998) 243-256.

[2] L.H. Kligman, F.J. Akin, A.M. Kligman, The contributions of UVA and UVB to connective tissue damage in hairless mice, J. Invest. Dermatol. 84 (1985) 272-276.

[3] J. Marto, L.F. Gouveia, B.G. Chiari, A. Paiva, V. Isaac, P. Pinto, P. Simões, A.J. Almeida, H.M. Ribeiro, The green generation of sunscreens: Using coffee industrial sub-products, Industrial Crops and Products 80 (2016) 93-100.

[4] R.M. Amin, S.A. Elfeky, T. Verwanger, B. Krammer, A new biocompatible nanocomposite as a promising constituent of sunscreens, Materials Science and Engineering C 63 (2016) 46-51.

[5] W-Y. Hsiao, S-J. Jiang, C-H. Feng, S-W. Wang, Y-L. Chen, Determining ultraviolet absorbents in sunscreen products by combining direct injection with micelle collapse on-line preconcentration capillary electrophoresis, J. Chromatogr. A 1383 (2015) 175-181.

[6] A. Morlando, D. Cardillo, T. Devers, K. Konstantinov, Titanium doped tin dioxide as potential UV filter with low photocatalytic activity for sunscreen products, Materials Letters 171 (2016) 289292.

[7] D.R. Sambandan, D. Ratner, Sunscreens: An overview and update, J AM ACAD DERMATOL, 64 (2011) 748-758.

[8] Camile L. Hexsel, Scott D. Bangert, Adelaide A. Hebert, Henry W. Lim, Current sunscreen issues: 2007 Food and Drug Administration sunscreen labelling recommendations and combination sunscreen/insect repellent products, J AM ACAD DERMATOL, 59 (2008) 316-323.

[9] A. Beeby, A.E. Jones, Photophysical properties of N-acetyl-menthyl anthranilate, Photochem. Photobiol., 64 (2001) 109-116.

[10] T.Karthick, V.Balachandran, S.Perumal, A.Nataraj, Spectroscopic studies, HOMO-LUMO and NBO calculations on monomer and dimer conformer of 5-nitrosalicylic acid, J.Mol.Struct, 1005 (2011) 192-201. 
[11] B.A.M.Corrêa, A.S.Gonçalves, A.M.T.de Souza, C.A.Freitas, L.M.Cabral. M.G.Albuquerque, H.C.Castro, E.P.dos Santos, C.R.Rodrigues, Molecular modeling studies of the structural, electronic, and UV absorption properties of benzophenone derivatives, J.Phys.Chem.A, 116 (2012) 10927-10933.

[12] Gaussian 09, Revision D.01, M. J. Frisch, G. W. Trucks, H. B. Schlegel, G. E. Scuseria, M. A. Robb, J. R. Cheeseman, G. Scalmani, V. Barone, B. Mennucci, G. A. Petersson, H. Nakatsuji, M. Caricato, X. Li, H. P. Hratchian, A. F. Izmaylov, J. Bloino, G. Zheng, J. L. Sonnenberg, M. Hada, M. Ehara, K. Toyota, R. Fukuda, J. Hasegawa, M. Ishida, T. Nakajima, Y. Honda, O. Kitao, H. Nakai, T. Vreven, J. A. Montgomery, Jr., J. E. Peralta, F. Ogliaro, M. Bearpark, J. J. Heyd, E. Brothers, K. N. Kudin, V. N. Staroverov, R. Kobayashi, J. Normand, K. Raghavachari, A. Rendell, J. C. Burant, S. S. Iyengar, J. Tomasi, M. Cossi, N. Rega, J. M. Millam, M. Klene, J. E. Knox, J. B. Cross, V. Bakken, C. Adamo, J. Jaramillo, R. Gomperts, R. E. Stratmann, O. Yazyev, A. J. Austin, R. Cammi, C. Pomelli, J. W. Ochterski, R. L. Martin, K. Morokuma, V. G. Zakrzewski, G. A. Voth, P. Salvador, J. J. Dannenberg, S. Dapprich, A. D. Daniels, Ö. Farkas, J. B. Foresman, J. V. Ortiz, J. Cioslowski, and D. J. Fox, Gaussian, Inc., Wallingford CT, 2009.

[13] A.R. ALLOUCHE, Gabedit - A graphical user interface for computational chemistry softwares, Journal of Computational Chemistry, 32 (2011) 174-182. 